\title{
Wandering Double-J Stent in the Retroperitoneum: A Case Report
}

\author{
Sridhar Panaiyadiyan, MS, Abhishek Shukla, MS, MCH, Brusabhanu Nayak, MS, MCH, \\ Prabhjot Singh, MS, MCH, DNB, and Ashish Kumar Saini, MS, MCH
}

\begin{abstract}
Background: Double-J (DJ) stent is an integral part of urologic practice. DJ stents can have numerous complications such as persistent irritative symptoms, encrustations, and up and down migrations within the pelvicaliceal system (PCS), but displacement outside the urinary tract is rare. We are presenting a unique case of DJ stent lying outside the PCS for more than a decade.

Case Presentation: A 46-year-old female presented with left flank pain and dysuria. She had undergone percutaneous nephrolithotomy in the left side 11 years ago. Imaging studies revealed a forgotten DJ stent with proximal part lying in the PCS with dense encrustations and the distal coil in the retroperitoneum at the level of the contralateral sacroiliac joint with the shaft crossing the midline at fourth lumbar vertebral level. The malpositioned forgotten stent was removed intact using the multimodal endourologic technique. During followup, the patient had prompt relief of symptoms and a preserved renal unit.

Conclusion: To the best of our knowledge, this is the first report of malpositioned and forgotten DJ stent for more than a decade with lower end lying near the opposite lower ureter managed effectively by an endourologic method.
\end{abstract}

Keywords: forgotten stent, malposition stent, Double-J stent, endourologic technique

\section{Introduction and Background}

T He Placement of Double-J (DJ) stent in the urinary tract is an integral part of urologic practice for various indications. However, DJ stent placement causes a myriad of complications ranging from trivial stent-related irritative lower urinary tract symptoms (LUTSs) to advanced renal failure in case of a forgotten stent. ${ }^{1}$ Timely removal prevents the long-term stent-related complications such as encrustations, migration, stent fracture, erosion, or fistulization. ${ }^{2}$ Although complications such as encrustations, proximal, and distal migrations within the pelvicaliceal system (PCS) are reported frequently in the literature, stent displacement outside the urinary tract is rare. Herein, we present a case of a forgotten DJ stent with proximal encrustation and distal segment lying in the retroperitoneum for more than a decade managed effectively by endourologic technique.

\section{Case Presentation}

History and physical examination

A 46-year-old female presented with left flank pain and burning micturition of 18 months duration. There was no history of LUTSs, fever, hematuria, or lithuria. Eleven years ago, the patient had undergone left percutaneous nephrolithotomy (PCNL) elsewhere for renal stone disease. However, she did not follow-up after the intervention with the primary surgeon. On presentation, the general and systemic examination did not reveal any abnormality.

\section{Diagnosis}

To our surprise, an upright kidney, urinary, and bladder radiograph (KUB) revealed a DJ stent with radiopaque shadow in the left renal coil and the distal coil lying near the contralateral sacroiliac joint with the shaft of DJ stent crossing the midline (Fig. 1A). The patient had normal renal function test, and a contrast-enhanced computed tomogram of KUB with urography done further showed a left hydronephrotic kidney with poor excretion as compared with the right renal unit. The upper end of the DJ stent lies inside the renal pelvis but with surrounding encrustations. However, the shaft of the DJ stent found emerging out of the upper ureter at the level of third lumbar (L3) vertebra, into the retroperitoneum, crossing the midline at the level of fourth lumbar (L4) vertebra, with the distal coil lying at the level of right sacral ala (Fig. 1B).

Department of Urology, All India Institute of Medical Sciences, New Delhi, India.

(C) Sridhar Panaiyadiyan et al. 2017; Published by Mary Ann Liebert, Inc. This is an Open Access article distributed under the terms of the Creative Commons Attribution License, which permits unrestricted use, distribution, and reproduction in any medium, provided the original work is properly cited. 

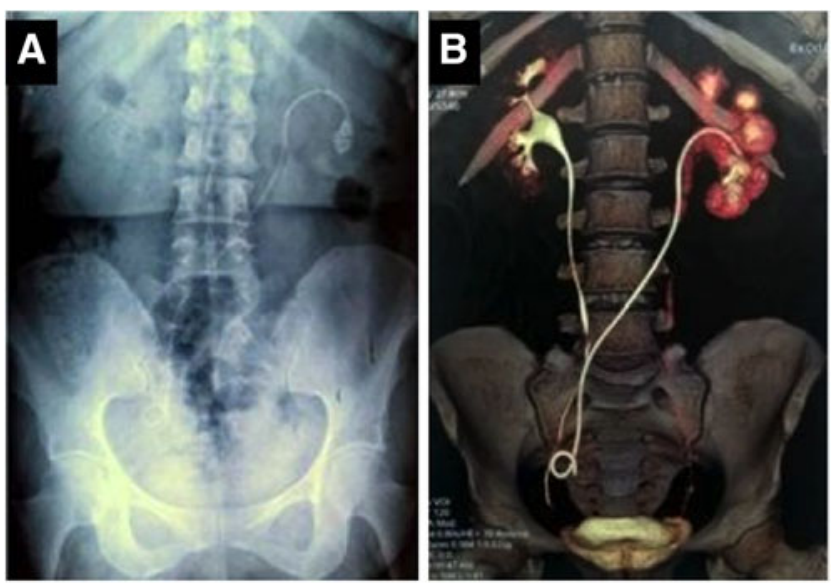

FIG. 1. (A) Upright KUB showing a DJ stent with radiopaque shadow in the left renal coil with the distal coil lying near the contralateral sacroiliac joint with the shaft of DJ stent crossing the midline at L4 lumbar vertebral level. (B) Three-dimensional reconstructed contrast-enhanced computed tomogram KUB with urography showing upper end of the DJ stent inside the renal pelvis with surrounding encrustations and the shaft of the DJ stent emerging out of the upper ureter at L3 vertebral level and crossing the midline at L4 vertebral level. DJ, Double-J; KUB, kidney urinary bladder radiograph.

A diuretic renogram revealed $20 \%$ split function of the left kidney with delayed clearance.

\section{Intervention}

The patient underwent cystoscopy with left retrograde pyelogram, which revealed injected contrast reaching the PCS (Fig. 2A). A semirigid ureteroscopy showed DJ stent extruding out of the upper ureter at the L3 vertebral level. In view of large encrustations around the renal coil of the DJ stent, we did a PCNL to dislodge the encrustations (Fig. 2B). A gentle traction applied on the upper coil with grasping forceps allowed intact retrieval of the whole of the DJ stent. A new DJ was placed under fluoroscopic guidance. The proximal third of the retrieved DJ stent lying inside the urinary tract stained dark, whereas the distal two-third lying in the retroperitoneum appeared clean and without encrustations (Fig. 2C).

\section{Outcome}

The postoperative recovery was uneventful and the patient underwent DJ stent removal after 6 weeks. At 3 months follow-up, diuretic renogram showed a $17 \%$ split function of the left kidney and nonobstructive clearance.

\section{Discussion and Literature Review}

Since its inception by Zimskind the placement of DJ stent became an integral part of urologic armamentarium. ${ }^{3}$ However, indwelling DJ stents is not free of complications. The most common complications are irritative LUTSs, suprapubic pain, and vesicoureteral reflux resulting in flank pain. ${ }^{2}$ Although proximal and distal migration of ureteral stent is a relatively common occurrence, displacement outside the urinary system is a rare phenomenon, caused by malposition of the DJ stent at the time of insertion or erosion of forgotten stent. ${ }^{4}$ In the present case, the intact DJ stent retrieved had two distinct zones of discoloration. The upper one-third of the stent stained dark, denoting the part with constant exposure to the urinary tract. In contrast, the distal two-thirds of the stent appeared clean, representing the part was unexposed to the urinary environment. The possible explanation would be that after the PCNL procedure, while attempting antegrade DJ stent, the surgeon would have perforated the ureter with the guidewire, over which the stent might be placed without image intensifier, resulting in malposition of the stent. The line of demarcation of the retrieved stent actually denotes the point at which the stent extrudes out of the urinary tract after perforating the ureteral wall (Fig. 2C).

As the distal end of the stent was lying in the retroperitoneum, the patient neither had irritative LUTSs because of bladder irritation nor had flank pain because of vesico ureteric reflux, the most common stent-related symptoms. This delayed her presentation for more than a decade until she developed the left flank pain and dysuria, leading to the detection of proximally encrusted, distally malpositioned, and forgotten stent in the current admission.

Management of the forgotten stent includes multimodal endourologic techniques through retrograde or antegrade approaches with or without auxiliary procedures such as extra or intracorporeal lithotripsy. ${ }^{5}$ Rarely a complete intraperitoneal displacement requires an open approach to retrieve the forgotten stent. ${ }^{4}$ In our case, partial malposition of the stent
FIG. 2. (A) Left retrograde pyelogram showing injected contrast reaching the PCS. (B) Intraoperative nephroscopic view showing encrustations of the proximal coil of the stent lying within the PCS. (C) The retrieved DJ stent with upper third stained dark (the part lying in the urinary tract) and lower twothirds appeared clean (the part lying in the retroperitoneum unexposed to urinary environment). PCS, pelvicaliceal system.
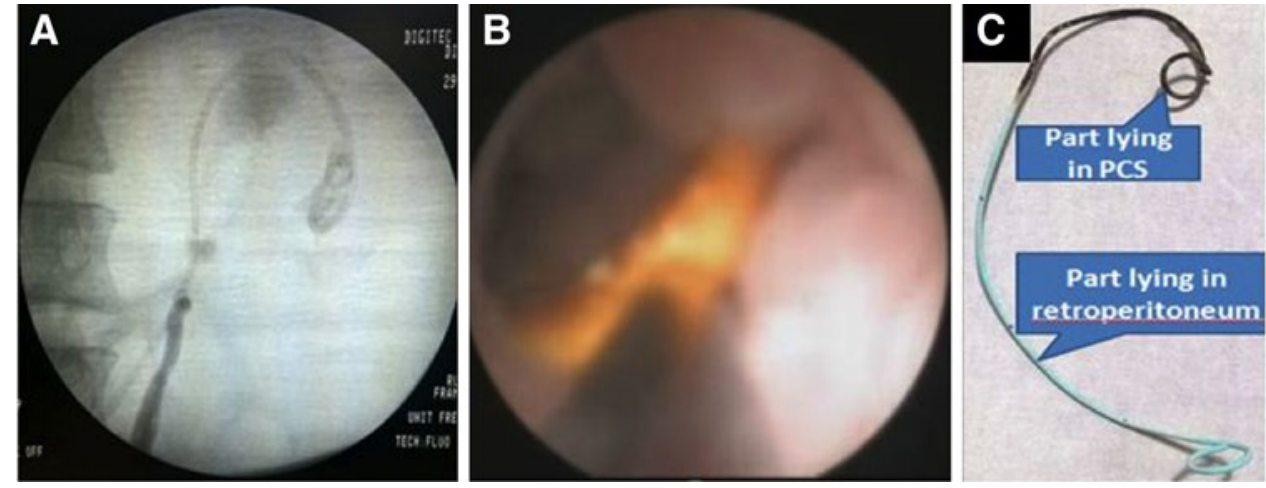
with the upper encrusted coil still in the PCS enabled us to completely retrieve the forgotten DJ stent through the antegrade approach.

\section{Conclusion}

To the best of our knowledge, this is the first report of malpositioned and forgotten DJ stent for more than a decade with lower end lying near the opposite lower ureter managed effectively by an endourologic method. As the famous saying "An ounce of prevention is worth a pound of cure," the placement of a DJ stent under the guidance of image intensifier, the first postoperative day plain roentgenogram, and the usage of computerized stent registry could prevent intraoperative stent malposition, early postoperative stent displacement, and late postoperative forgotten stent, respectively, at different timelines.

\section{Disclosure Statement}

No competing financial interests exist.

\section{References}

1. Sohrab A, Aneesh S, Sureka SK, Varun M, Nitesh P, Manoj $\mathrm{K}$, et al. Forgotten reminders: An experience with managing 28 forgotten Double-J stents and management of related complications. Indian J Surg 2015;77(S3):1165-1171.

2. Dyer RB, Chen MY, Zagoria RJ, Regan JD, Hood CG, Kavanagh PV. Complications of ureteral stent placement. Radiographics 2002;22:1005-1022.

3. Zimskind PD, Fetter TR, Wilkerson JL. Clinical use of longterm indwelling silicone rubber ureteral splints inserted cystoscopically. J Urol 1967;97:840-844.
4. Turri FM, Manassero F, Mogorovich A, De Maria M, Falleni A, Selli C. Complete intraperitoneal displacement of a double J stent: A first case. Arch Ital Urol E Androl 2015;87:95.

5. Singh V, Srinivastava A, Kapoor R, Kumar A. Can the complicated forgotten indwelling ureteric stents be lethal? Int Urol Nephrol 2005;37:541-546.

Address correspondence to:

Brusabhanu Nayak, MS, MCH

Department of Urology

All India Institute of Medical Sciences

Ansari Nagar

New Delhi 110029

India

E-mail: brusabhanu@gmail.com

Cite this article as: Panaiyadiyan S, Shukla A, Nayak B, Singh P, Saini AK (2017) Wandering Double-J stent in the retroperitoneum: a case report, Journal of Endourology Case Reports 3:1, 189-191, DOI: 10.1089/ cren.2017.0126.

$$
\begin{aligned}
\text { DJ } & =\text { Double-J } \\
\text { KUB } & =\text { kidney, ureter, and bladder radiograph } \\
\text { LUTSs } & =\text { lower urinary tract symptoms } \\
\text { PCNL } & =\text { percutaneous nephrolithotomy } \\
\text { PCS } & =\text { pelvicaliceal system }
\end{aligned}
$$

